\title{
A clinicopathological study of fatal chronic airways obstruction
}

\author{
K . W . M . S C O T T ${ }^{1}$ \\ Pathology Department, University of Sheffield
}

\begin{abstract}
Scott, K. W. M. (1976). Thorax, 31, 693-701. A clinicopathological study of fatal chronic airways obstruction. A clinicopathological study of 21 patients who died as a result of chronic airways obstruction was carried out. Thirteen patients had been in right ventricular failure for at least one year before death and the other eight patients did not have right ventricular failure. The patients with long-standing right ventricular failure died at a younger age, on average, than those without failure. There were no significant quantitative differences between the two groups in the length of history of chest disease, blood gas estimations, respiratory function tests or degree of polycythaemia. The group with right ventricular failure had significantly larger mean right and left ventricular weights than the group without failure, but there were no significant differences in amounts of emphysema, size of bronchial mucous glands, proportion of small airways lumen in the lung or number of thick-walled peripheral lung vessels between the two groups.

The findings did not support the division of this series of patients, with fatal chronic airways obstruction, into two distinct groups broadly defined as 'emphysematous' and 'bronchitic', either clinically or pathologically. A history of right ventricular failure correlated well with the finding of right ventricular hypertrophy at necropsy. Electrocardiographic evidence of right ventricular hypertrophy was found to correspond with the size of the right ventricle at necropsy in $66 \%$ of cases. The radiographic diagnosis of emphysema proved an accurate assessment when compared to the necropsy findings, and radiographic estimations of right ventricular enlargement were accurate in $65 \%$ of cases.

Histological evidence of acute bronchitis was present in 20 of the 21 patients $(95 \%)$, and five patients showed histological evidence of minor pulmonary thromboembolism. Ten patients in the series showed an increase in the weight of the left ventricle as well as the right ventricle.
\end{abstract}

The fact that chronic pulmonary disease may result in heart failure was recognized by Laënnec in 1819. Several authors have attempted to divide patients with obstructive airways disease into two broad groups, one known as 'bronchitic' who usually have heart failure, and the other known as 'emphysematous' who do not have such a high incidence of heart failure (Ogilvie, 1959; Richards, 1960; Fletcher et al., 1963; Burrows et al., 1964; Briscoe and Nash, 1965). Burrows et al. (1966) found that chronic or recurrent heart failure occurred in what they termed type B disease where there was absent or mild emphysema. They 'Present address: Pathology Department, The Royal Hospital,
Wolverhampton. also included a type $\mathrm{X}$ disease for patients who did not fulfil the criteria for type A (severe emphysema) or type B disease. Heath, Brewer, and Hicken (1968) suggested that right ventricular hypertrophy was more common in centrilobular than in panlobular emphysema. The aim of the present study was to determine from clinical and pathological data whether there are two distinct groups of patients who die from obstructive airways disease and also to establish whether quantitative differences existed in the airways or alveoli. The study also yielded information on the accuracy of clinical examination and tests in predicting the presence of right ventricular hypertrophy and on the nature of the process in the lungs which precipitated death in these patients. 


\section{MATERIAL AND METHODS}

Twenty-one known cases of chronic airways obstruction which had been investigated in the Department of Medicine at Sheffield Royal Hospital and subsequently came to necropsy were studied.

All the patients had a history of cough and daily sputum production for at least three months in the year, for two or more successive years (Ciba Guest Symposium Report, 1959). They all had dyspnoea which was sufficiently severe to make them stop while walking on the level and they all had a normal systemic blood pressure.

The case records were examined to determine the total length of history of chest disease and also the length of time the patients had been in cardiac failure. This was defined as the presence of ankle oedema and raised jugular venous pressure (greater than $2 \mathrm{~cm}$ of water above the manubriosternal junction at an angle of $45^{\circ}$ ) (Anderson et al., 1973). Electrocardiograms were performed during the terminal illness. These were examined for evidence of right ventricular hypertrophy judged by a dominant $R$ or an inverted $T$ wave in V1-3 or a dominant $R$ in aVR and dominant $S$ in V5. The presence of $P$ pulmonale was also ascertained and was considered to be present if the $\mathbf{P}$ wave was greater than $2 \mathrm{~mm}$ in $\mathbf{S} 2$ (Anderson et al., 1973). Chest radiographs were examined during the terminal illness for signs of emphysema (judged by the degree of peripheral pruning of vessels and the presence of visible bullae) and cardiac enlargement. Before the terminal admission, in a quiescent phase of the disease, forced expiratory volume at one second $\left(\mathrm{FEV}_{1}\right)$ and forced vital capacity had been determined with a Poulton spirometer. Also at this time total lung volume and residual volume were determined by a closed-circuit helium dilution technique. During the terminal illness arterial blood gas estimations were performed using EIL electrodes, and the haemoglobin and packed cell volume were determined on venous blood.

At necropsy the hearts were fixed, after examination of the coronary arteries and valves, in $10 \%$ formol saline for $72-96$ hours and were then dissected according to the method of Fulton, Hutchinson, and Jones (1952). None of the cases showed gross coronary artery atheroma, valvular disease, myocardial infarction or fibrosis. The right ventricle and the left ventricle plus the septum were weighed separately, and the ratio of the left to right ventricular weight was calculated. After removal from the thorax the lungs were distended with $10 \%$ formol saline introduce $\frac{\text { के }}{6}$ by a catheter into the main bronchus at a pressure of $30 \mathrm{~cm}$ of water, using a modification of the apparatus designed by Heard (1969). In 12 cases both lungs were distended while in the other nine only the left lung was examined. After fixations in this state samples of the bronchial tree werẹ taken from three sites in the left lung: (a) the lef $t$ main bronchus just proximinal to its bifurcation $\omega_{\sigma}$ (b) the bronchus to the basal segments (the bron $\overrightarrow{2}$ chus immediately below the origin of the bronchus to the apical segment of the lower lobe), and (c) the bronchus to the inferior segment of the lingula, $5 \mathrm{~mm}$ beyond its origin (Restrepo ande Heard, 1963). Transverse blocks were embeddeb in paraffin and $5 \mu$ sections were stained by haematoxylin and eosin and periodic acid Schiff $\vec{\square}$ after which they were projected at a magnificationg of 20 diameters on to a point-counting grid (the points were at angles of equilateral triangles of side $0.6 \mathrm{~cm}$ ) (Dunnill, Massarella, and Anderson, $\frac{\mathscr{}}{-}$ 1969). The number of points falling on bronchiate mucous glands was expressed as a percentage of̂ the total number of points falling on all tissues, and a mean was taken of the values for each of the three sites. The lungs were then sliced in the parasagittal plane at $1 \mathrm{~cm}$ intervals, and aften barium sulphate impregnation (Heard, 1958) threes or four slices were examined to determine the amount and type of emphysema present. This was done using a plastic grid and a point-counting technique (Dunnill, 1962). The type of tissue under each point in the grid was noted and count was made of the following components in each slice: panlobular emphysema, centrilobula emphysema, normal lung, and non-parenchyma (blood vessels, bronchi, pleura, etc). The amoun of each type of emphysema was expressed as percentage of the total number of points counteक and a mean was taken of the three or four slices examined. Where both lungs were examined mean was taken of the amount of emphysema iro each lung. Using a stratified random technique (Dunnill, 1968), six blocks of lung were takery from each case. These were approximately $2.5 \times 1.5 \times 0.5 \mathrm{~cm}$ in size, and after paraffin em^ bedding and sectioning they were stained with haematoxylin and eosin. The relative proportions of small airways lumen of diameter less thar $2 \mathrm{~mm}$ were calculated in these sections using are eyepiece graticule with 25 equidistant points Each point was scored as either small airways lumen or other lung tissue, and the proportion of small airways lumen $(\mathrm{Q})$ in the lung was expres $\overrightarrow{\mathbb{D}}$ sed as a percentage of the total number of points 
counted (Matsuba and Thurlbeck, 1971). It was assumed that shrinkage due to fixation and processing was equal in small airways and in the remainder of the lung tissue, and $Q$ was not corrected for the degree of inflation as this has been found not to be of major significance (Matsuba and Thurlbeck, 1972).

Sections from the same six random blocks of lung tissue were also stained by the Weigert-vanGieson method. The whole section was examined with a $\times 25$ objective lens to determine the number of small vessels of less than $100 \mu$ in diameter which had a double elastic lamina with a layer of muscle between the laminae. This was done using a modification of the technique of Hunter et al. (1974). All small vessels with a definite elastic coat were counted, and the number of them which had a double elastic layer was expressed as a percentage of the total number of vessels counted. Small venules were included with the arterioles as it is very difficult to distinguish these without seeing them join an artery or vein (Brenner, 1935). Vessels of diameter less than $100 \mu$ were counted since this is the upper limit of size of pulmonary arterioles found by Brenner (1935). The criteria of Hunter et al. (1974) were used to distinguish thick-walled peripheral lung vessels from normal small vessels. A double elastic lamina was said to be present when two laminae, with a space between them, were visible for at least half the diameter in cross section, or half the length of the wall in longitudinal section. Twenty random sections of lung were examined to establish the presence of acute suppurative bronchitis and bronchiolitis. This was defined as an exudate of acute inflammatory cells mixed with mucus which appeared to fill small airways. The sections were also examined for the presence of pneumonia and for evidence of thromboembolism in the small pulmonary arteries. None of the cases showed major thromboembolism in the large pulmonary arteries.

\section{RESULTS}

The 21 patients were divided into two groups according to the presence or absence of the clinical signs of right ventricular failure. Thirteen patients had been in right ventricular failure for at least one year before death, and their clinical details are shown in Table I. The other eight patients had never shown signs of right ventricular failure during life or only developed them during the terminal episode of the disease. The clinical details of these patients are shown in Table II. By the criteria of Burrows et al. (1966) all the patients had airways obstruction. The FEV 1 was less than 1.251 in all the patients where it was measured, and the $\mathrm{FEV}_{1}: \mathrm{FVC}$ ratio was $50 \%$ or less in all patients except case 66 in whom it was $55.5 \%$.

T A B L E I

CLINICAL DETAILS OF 13 PATIENTS WITH RIGHT VENTRICULAR FAILURE

\begin{tabular}{|c|c|c|c|c|c|c|c|c|c|c|c|c|c|c|}
\hline Case & $\begin{array}{c}\text { Age at } \\
\text { Death } \\
\text { (yr) }\end{array}$ & Sex & $\begin{array}{c}\text { History } \\
\text { (yr) }\end{array}$ & $\begin{array}{c}\text { Duration } \\
\text { of RV } \\
\text { Failure } \\
\text { (yr) }\end{array}$ & ECG & CXR & $\underset{\text { (I) }}{\text { FEV }_{1}}$ & $\begin{array}{c}\text { FVC } \\
\text { (l) }\end{array}$ & $\begin{array}{l}\text { RV/ } \\
\text { TLC } \\
(\%)\end{array}$ & $\underset{(\mathrm{mmHg})}{\mathrm{PaO}_{2}}$ & $\underset{\left(\mathrm{mmHg}^{2}\right)}{\mathrm{PaCO}_{2}}$ & pH & $\begin{array}{c}\text { Haemo- } \\
\text { globin } \\
\text { (g/dl) }\end{array}$ & $\begin{array}{l}\text { PCV } \\
(\%)\end{array}$ \\
\hline 16 & 62 & $\mathbf{F}$ & 10 & 4 & \multirow{8}{*}{$\begin{array}{l}\text { PP } \\
\text { RVH } \\
\text { RVH } \\
\text { NAD } \\
\text { PP } \\
\text { RVH } \\
\text { PP } \\
\text { RVH } \\
\text { PP } \\
\text { RVH } \\
\text { PP } \\
\text { RVH } \\
\text { NAD } \\
\text { NAD } \\
\text { PP } \\
\text { PP } \\
\text { RVH } \\
\text { PP } \\
\text { PP } \\
\text { RVH }\end{array}$} & EH & 0.50 & $1 \cdot 0$ & 61 & 38 & 63 & $7 \cdot 35$ & $16 \cdot 5$ & 61 \\
\hline $\begin{array}{l}21 \\
27 \\
30\end{array}$ & $\begin{array}{l}47 \\
76 \\
51\end{array}$ & $\begin{array}{l}\mathbf{M} \\
\mathbf{M} \\
\mathbf{M}\end{array}$ & $\begin{array}{l}12 \\
10 \\
17\end{array}$ & $\begin{array}{l}5 \\
2 \\
1 \cdot 5\end{array}$ & & $\begin{array}{l}\mathbf{H} \\
\mathbf{E} \\
\mathbf{H}\end{array}$ & $\begin{array}{l}0.60 \\
0.25 \\
0.50\end{array}$ & $\begin{array}{l}1 \cdot 8 \\
0.85 \\
2 \cdot 1\end{array}$ & $\overline{53}$ & $\begin{array}{l}29 \\
44 \\
26\end{array}$ & $\begin{array}{l}70 \\
62 \\
68\end{array}$ & $\begin{array}{l}7 \cdot 32 \\
7 \cdot 36 \\
7 \cdot 44\end{array}$ & $\frac{14 \cdot 7}{14 \cdot 8}$ & $\begin{array}{l}57 \\
57 \\
-\end{array}$ \\
\hline 34 & 60 & $\mathbf{M}$ & 30 & $1 \cdot 5$ & & E & $0 \cdot 30$ & $1 \cdot 4$ & - & 38 & 78 & $7 \cdot 30$ & $16 \cdot 7$ & 56 \\
\hline 45 & 61 & $\mathbf{M}$ & 13 & 1 & & EH & 0.50 & $1 \cdot 8$ & - & 36 & 86 & $7 \cdot 28$ & $20 \cdot 6$ & 70 \\
\hline 46 & 38 & F & 20 & $2 \cdot 5$ & & EH & 0.40 & $1 \cdot 75$ & 65 & 27 & 72 & $7 \cdot 32$ & $16 \cdot 7$ & - \\
\hline $\begin{array}{l}49 \\
51 \\
66 \\
70\end{array}$ & $\begin{array}{l}66 \\
64 \\
70 \\
49\end{array}$ & $\begin{array}{l}\mathbf{M} \\
\mathbf{M} \\
\mathbf{M} \\
\mathbf{F}\end{array}$ & $\begin{array}{r}20 \\
15 \\
20 \\
3\end{array}$ & $\begin{array}{r}11 \\
1 \\
6 \\
1\end{array}$ & & $\begin{array}{l}\mathrm{EH} \\
\mathrm{EH} \\
\overline{\mathrm{EH}}\end{array}$ & $\begin{array}{l}0.60 \\
0.45 \\
1 \cdot 0 \\
0.30\end{array}$ & $\begin{array}{l}1 \cdot 8 \\
2.3 \\
1.8 \\
1.5\end{array}$ & $\begin{array}{l}35 \\
65 \\
-\end{array}$ & $\begin{array}{l}42 \\
45 \\
54 \\
41\end{array}$ & $\begin{array}{l}54 \\
59 \\
45 \\
53\end{array}$ & $\begin{array}{l}7 \cdot 36 \\
7 \cdot 39 \\
7 \cdot 50 \\
7 \cdot 50\end{array}$ & $\begin{array}{l}17 \cdot 8 \\
11 \cdot 3 \\
16 \cdot 6 \\
12 \cdot 9\end{array}$ & $\begin{array}{l}\frac{61}{50} \\
45\end{array}$ \\
\hline $\begin{array}{l}73 \\
80\end{array}$ & $\begin{array}{l}61 \\
63\end{array}$ & $\begin{array}{l}\mathbf{M} \\
\mathbf{M}\end{array}$ & $\begin{array}{l}10 \\
10\end{array}$ & $\begin{array}{l}1 \cdot 5 \\
1.5\end{array}$ & & $\begin{array}{l}\text { EH } \\
\text { EH }\end{array}$ & $\begin{array}{l}0.53 \\
0.60\end{array}$ & $\begin{array}{l}1.46 \\
1.58\end{array}$ & $\begin{array}{l}59 \\
62\end{array}$ & $\begin{array}{l}37 \\
40\end{array}$ & $\begin{array}{l}63 \\
62\end{array}$ & $\begin{array}{l}7 \cdot 31 \\
7 \cdot 39\end{array}$ & $\begin{array}{l}22 \cdot 2 \\
17 \cdot 4\end{array}$ & $\begin{array}{l}69 \\
59\end{array}$ \\
\hline$\underset{\text { SD }}{\text { Mean }}$ & $\begin{array}{l}59 \\
10.2\end{array}$ & & $\begin{array}{r}14 \cdot 6 \\
6.8\end{array}$ & $\begin{array}{l}3 \\
2 \cdot 8\end{array}$ & & . & $\begin{array}{l}0.50 \\
0.17\end{array}$ & $\begin{array}{l}1 \cdot 63 \\
0.38\end{array}$ & $\begin{array}{l}55 \cdot 7 \\
10.0\end{array}$ & $\begin{array}{r}38.2 \\
7 \cdot 7\end{array}$ & $\begin{array}{l}64 \cdot 1 \\
10 \cdot 8\end{array}$ & $\begin{array}{l}7 \cdot 37 \\
0 \cdot 20\end{array}$ & $\begin{array}{r}16 \cdot 5 \\
2.9\end{array}$ & $\begin{array}{r}58 \cdot 5 \\
7.6\end{array}$ \\
\hline
\end{tabular}

ECG = electrocardiogram; PP = P pulmonale; RVH = right ventricular hypertrophy (see text); CXR =chest $x$-ray; $E=$ emphysema (peripheral pruning of vessels and bullae); $\mathbf{H}=$ enlargement of right heart; $F E V_{1}=$ forced expiratory volume at one second; $F$ VC=forced vital capacity; $\mathbf{R V}=$ residual volume; TLC=total lung capacity; $\mathrm{PaO}_{2}=$ arterial oxygen tension; $\mathrm{PaCO}_{2}=$ arterial carbon dioxide tension; $\mathrm{PCV}=$ packed cell volume (haematocrit); NAD = normal. 
T A B L E I I

CLINICAL DETAILS OF THE EIGHT PATIENTS WITHOUT RIGHT VENTRICULAR FAILURE

\begin{tabular}{|c|c|c|c|c|c|c|c|c|c|c|c|c|c|}
\hline Case & $\begin{array}{c}\text { Age at } \\
\text { Death } \\
(y r)\end{array}$ & Sex & $\begin{array}{c}\text { History } \\
\text { (yr) }\end{array}$ & ECG & CXR & $\underset{\text { (1) }}{\mathrm{FEV}_{1}}$ & $\begin{array}{c}\text { FVC } \\
\text { (l) }\end{array}$ & $\begin{array}{l}\text { RV/ } \\
\text { TLC } \\
\%\end{array}$ & $\underset{(\mathrm{mmHg})}{\mathrm{PaO}_{2}}$ & $\begin{array}{c}\mathrm{PaCO}_{2} \\
(\mathrm{mmHg})\end{array}$ & $\mathrm{pH}$ & $\begin{array}{l}\text { Haemo- } \\
\text { globin } \\
\text { (g/dl) }\end{array}$ & $\begin{array}{c}\text { PCV } \\
\%\end{array}$ \\
\hline 9 & 69 & $\mathbf{M}$ & 25 & \multirow{6}{*}{$\begin{array}{l}\text { PP } \\
\text { RVH } \\
\text { NAD } \\
\text { PP } \\
\text { RVH } \\
\text { PP } \\
\text { RVH } \\
\text { PP } \\
\text { RVH } \\
\text { PP } \\
\text { RVH } \\
\text { PP } \\
\text { PP }\end{array}$} & EH & 0.35 & $1 \cdot 0$ & 64 & 38 & 97 & $7 \cdot 24$ & $16 \cdot 0$ & 50 \\
\hline $\begin{array}{l}15 \\
19\end{array}$ & $\begin{array}{l}70 \\
74\end{array}$ & $\begin{array}{l}\mathrm{F} \\
\mathrm{M}\end{array}$ & $\begin{array}{l}20 \\
16\end{array}$ & & - & - & - & - & $\begin{array}{l}32 \\
45\end{array}$ & $\begin{array}{r}110 \\
53\end{array}$ & $\begin{array}{l}7 \cdot 20 \\
7 \cdot 42\end{array}$ & $\begin{array}{l}16 \cdot 5 \\
17 \cdot 9\end{array}$ & $\overline{62}$ \\
\hline 20 & 60 & $\mathbf{M}$ & 9 & & E & 0.55 & 1.45 & 66 & 26 & 53 & $7 \cdot 39$ & $16 \cdot 5$ & 57 \\
\hline 50 & 64 & $\mathbf{M}$ & 5 & & $\mathbf{E H}$ & $0 \cdot 30$ & $1 \cdot 6$ & - & 36 & 45 & $7 \cdot 35$ & $14 \cdot 4$ & - \\
\hline 68 & 68 & $\mathbf{M}$ & 3 & & - & - & - & - & 21 & 85 & $7 \cdot 23$ & - & 59 \\
\hline $\begin{array}{l}72 \\
74\end{array}$ & $\begin{array}{l}68 \\
70\end{array}$ & $\begin{array}{l}\mathbf{M} \\
\mathbf{M}\end{array}$ & $\begin{array}{l}10 \\
50\end{array}$ & & $\begin{array}{l}\mathrm{E} \\
\mathrm{EH}\end{array}$ & $\begin{array}{l}0.77 \\
0.44\end{array}$ & $\begin{array}{l}2 \cdot 28 \\
1 \cdot 14\end{array}$ & $\begin{array}{l}64 \\
66\end{array}$ & $\begin{array}{l}66 \\
55\end{array}$ & $\begin{array}{l}52 \\
40\end{array}$ & $\begin{array}{l}7 \cdot 39 \\
7 \cdot 44\end{array}$ & $\begin{array}{l}15 \cdot 0 \\
15 \cdot 2\end{array}$ & $\overline{47}$ \\
\hline $\begin{array}{c}\text { Mean } \\
\text { SD }\end{array}$ & $\begin{array}{r}67 \cdot 8 \\
4 \cdot 8\end{array}$ & & $\begin{array}{l}17 \cdot 2 \\
15 \cdot 1\end{array}$ & & & $\begin{array}{l}0.48 \\
0.17\end{array}$ & $\begin{array}{l}1.49 \\
0.50\end{array}$ & $\begin{array}{r}65 \cdot 0 \\
1 \cdot 1\end{array}$ & $\begin{array}{l}39 \cdot 8 \\
16 \cdot 1\end{array}$ & $\begin{array}{r}66 \cdot 8 \\
9 \cdot 2\end{array}$ & $\begin{array}{l}7 \cdot 33 \\
0 \cdot 10\end{array}$ & $\begin{array}{c}15 \cdot 9 \\
1 \cdot 14\end{array}$ & $\begin{array}{l}55 \\
6 \cdot 2\end{array}$ \\
\hline
\end{tabular}

Of the 11 patients who had lung volume studies, 10 had RV/TLC ratios greater than $50 \%$, and one (case 49) had a value of $35 \%$. Analysis of the arterial blood gas tensions showed that all the patients had respiratory failure $\left(\mathrm{PaO}_{2}<75 \mathrm{mmHg}\right.$ and/or $\mathrm{PaCO}_{2}>47 \mathrm{mmHg}$ ) (Ciba Guest Symposium Report, 1959). Thirteen patients had a haemoglobin or haematocrit which could be considered to be in the polycythaemic range (haemoglobin $>18.0 \mathrm{~g} / \mathrm{dl}$ in male or $>16.4 \mathrm{~g} / \mathrm{dl}$ in female; PCV $>54 \%$ in male or $>47 \%$ in female) (De Gruchy, 1964). Nine of these patients were in the group with right ventricular failure and four were not. There was no significant difference between the number of polycythaemic patients in failure and the number not in failure $\left(\chi^{2}=0.79, \mathrm{P}=\right.$ $>0 \cdot 30$ ). The mean values for each of the two groups were compared for all the clinical parameters using Student's $t$ test (Hill, 1971). The results of these comparisons are shown in Table III. It will be seen that the mean age at death was significantly lower in the group with right ventricular failure but that there were no other significant differences in respiratory function tests,

T A B L E I I I

COMPARISON OF MEAN VALUES OF CLINICAL PARAMETERS IN THE TWO GROUPS $(t$ TEST)

\begin{tabular}{l|ccc}
\hline \multicolumn{1}{c|}{ Parameter } & $t$ & P & \\
\hline Age at death & 2.26 & $<0.05$ & Significant \\
Length of history & 0.55 & $>0.50$ & \\
FEV FVC $_{\text {RV:TLC (\%) }}$ & 0.22 & $>0.80$ & \\
PaO $_{2}$ & 0.63 & $>0.50$ & $>0.10$ \\
Paco $_{2}$ & 1.81 & $>0.70$ \\
pH & 0.33 & $>0.50$ \\
Haemoglobin & 0.58 & $>0.50$ \\
Packed cell volume & 0.57 & $>0.60$ \\
& 0.50 & $>0.30$ & \\
\hline
\end{tabular}

arterial blood gases or haematological parameters $\overbrace{\overparen{\Phi}}$ between the two groups.

The pathological details for the 13 patients with right ventricular failure are shown in Table IV. $\overrightarrow{0}$ These include heart weights, amounts of emphysema, bronchial mucous gland sizes, proportion of small airways lumen in the lung, and pro-o portion of thick-walled peripheral lung vessels. Similar details for the eight patients who did noto have right ventricular failure are shown in Table $\frac{\varnothing}{\varnothing}$ $V$. The mean values for each of the two groups $\cong$ were compared using Student's $t$ test, and the re- $\overrightarrow{0}$ sults of this are shown in Table VI. The group with right ventricular failure had a mean left? ventricular weight of $195.3 \mathrm{~g}$ and this was significantly greater than the mean of the group whos did not have failure $(155.2 \mathrm{~g})$. There was also a significantly greater mean right ventricular weight $x$ $(132.9 \mathrm{~g})$ in the group with right ventricular $\frac{3}{3}$ failure than in the non-failure group (70.8 g).

The ratio of the weight of the left to the right 3 ventricle was significantly smaller in the right ${ }_{0}$ ventricular failure group. There were no significant differences between the two groups in theo mean amount of emphysema (total or either type), the size of the bronchial mucous glands, the pro-cr portion of small airways lumen or the number of thick-walled peripheral lung vessels. Five patientsn had normal right ventricular weights $(<65 \mathrm{~g})_{\mathrm{O}}^{\omega}$ (Fulton et al., 1952) and all of these were in the group who did not have right ventricular failure. All the patients who had clinical evidence of rightos ventricular failure had right ventricular hyper- 0 trophy $(>80 \mathrm{~g})$. Left ventricular hypertrophyö $(>225 \mathrm{~g}$ ) was present in only one case (66), butक्ष there were a further nine cases in whom the left ${ }_{0}$ ventricular weight was greater than the accepted $\frac{\text { 응 }}{\circ}$ 
T A B L E I V

PATHOLOGICAL DETAILS OF 13 PATIENTS WITH RIGHT VENTRICULAR FAILURE

\begin{tabular}{|c|c|c|c|c|c|c|c|c|c|c|c|}
\hline \multirow[b]{2}{*}{ Case } & \multicolumn{2}{|c|}{$\begin{array}{l}\text { Ventricular } \\
\text { Weight } \\
\text { (g) }\end{array}$} & \multirow[b]{2}{*}{ LV/RV } & \multirow{2}{*}{$\begin{array}{c}\text { Total } \\
\text { Amount of } \\
\text { Emphysema } \\
(\%)\end{array}$} & \multirow{2}{*}{$\begin{array}{c}\text { Centri- } \\
\text { lobular } \\
\text { Emphysema } \\
(\%)\end{array}$} & \multirow{2}{*}{$\begin{array}{c}\text { Panlobular } \\
\text { Emphysema } \\
(\%)\end{array}$} & \multirow{2}{*}{$\begin{array}{c}\text { Bronchial } \\
\text { Mucous } \\
\text { Gland Size } \\
(\%)\end{array}$} & \multirow{2}{*}{$\begin{array}{c}\text { Small } \\
\text { Airways } \\
\text { Lumen } \\
(\%)\end{array}$} & \multirow{2}{*}{$\begin{array}{c}\text { Thick-walled } \\
\text { Peripheral } \\
\text { Lung } \\
\text { Vessels } \\
(\%)\end{array}$} & \multirow{2}{*}{$\underset{\text { Bcute }}{\text { Aconchitis }}$} & \multirow{2}{*}{$\begin{array}{l}\text { Pulmonary } \\
\text { Thrombo- } \\
\text { embolism }\end{array}$} \\
\hline & Left & Right & & & & & & & & & \\
\hline $\begin{array}{l}16 \\
21 \\
27 \\
30 \\
34 \\
45 \\
46 \\
49 \\
51 \\
66 \\
70 \\
73 \\
80\end{array}$ & $\begin{array}{l}180 \\
209 \\
196 \\
213 \\
160 \\
200 \\
203 \\
205 \\
175 \\
250 \\
110 \\
213 \\
225\end{array}$ & $\begin{array}{r}140 \\
115 \\
88 \\
152 \\
95 \\
160 \\
199 \\
140 \\
110 \\
110 \\
120 \\
150 \\
150\end{array}$ & $\begin{array}{l}1 \cdot 3 \\
1 \cdot 8 \\
2 \cdot 2 \\
1.4 \\
1 \cdot 7 \\
1 \cdot 2 \\
1.0 \\
1 \cdot 5 \\
1.6 \\
2.3 \\
0.9 \\
1 \cdot 4 \\
1 \cdot 5\end{array}$ & $\begin{array}{l}31 \\
21^{*} \\
44^{*} \\
55^{*} \\
21 \\
45 \\
62^{*} \\
28 \\
44^{*} \\
7^{*} \\
88^{*} \\
95^{*} \\
37^{*}\end{array}$ & $\begin{array}{r}30 \\
20 \\
14 \\
8 \\
10 \\
16 \\
3 \\
28 \\
3 \\
7 \\
0 \\
0 \\
9\end{array}$ & $\begin{array}{r}1 \\
1 \\
30 \\
47 \\
11 \\
29 \\
59 \\
0 \\
41 \\
0 \\
88 \\
95 \\
28\end{array}$ & $\begin{array}{l}19.7 \\
18.6 \\
12.8 \\
14.1 \\
26.0 \\
19.7 \\
20.5 \\
15.6 \\
14.0 \\
11.5 \\
11 \cdot 2 \\
21.5 \\
11.5\end{array}$ & $\begin{array}{l}1.57 \\
1.15 \\
0.13 \\
0.86 \\
0.61 \\
0.74 \\
0.68 \\
0.49 \\
1.01 \\
1.55 \\
0.31 \\
0.22 \\
0.65\end{array}$ & $\begin{array}{l}10.6 \\
16.9 \\
19.3 \\
19.6 \\
13.6 \\
22.5 \\
17.5 \\
18.6 \\
13.5 \\
20.7 \\
17.3 \\
15.7 \\
15.9\end{array}$ & $\begin{array}{l}+ \\
+ \\
+ \\
+ \\
+ \\
+ \\
+ \\
+ \\
+ \\
+ \\
+ \\
+ \\
+\end{array}$ & $\begin{array}{l}\overline{+} \\
\overline{-} \\
- \\
- \\
- \\
\overline{-} \\
\pm \\
\pm \\
-\end{array}$ \\
\hline$\underset{\text { SD }}{\text { Mean }}$ & $\begin{array}{r}195 \cdot 3 \\
34 \cdot 2\end{array}$ & $\begin{array}{r}132 \cdot 9 \\
30 \cdot 5\end{array}$ & $\begin{array}{l}1 \cdot 52 \\
0 \cdot 41\end{array}$ & $\begin{array}{l}44 \cdot 4 \\
25 \cdot 6\end{array}$ & $\begin{array}{r}11 \cdot 4 \\
9 \cdot 8\end{array}$ & $\begin{array}{l}33 \cdot 0 \\
31 \cdot 1\end{array}$ & $\begin{array}{r}16 \cdot 6 \\
4 \cdot 6\end{array}$ & $\begin{array}{l}0.77 \\
0.45\end{array}$ & $\begin{array}{r}17 \cdot 0 \\
3 \cdot 3\end{array}$ & & \\
\hline
\end{tabular}

Mean of right and left lungs.

T A B L E V

PATHOLOGICAL DETAILS OF THE EIGHT PATIENTS WITHOUT RIGHT VENTRICULAR FAILURE

\begin{tabular}{|c|c|c|c|c|c|c|c|c|c|c|c|}
\hline \multirow[b]{2}{*}{ Case } & \multicolumn{2}{|c|}{$\begin{array}{c}\text { Ventricular } \\
\text { Weight } \\
\text { (g) }\end{array}$} & \multirow[b]{2}{*}{ LV/RV } & \multirow{2}{*}{$\begin{array}{l}\text { Total } \\
\text { Amount of } \\
\text { Emphysema } \\
(\%)\end{array}$} & \multirow{2}{*}{$\begin{array}{c}\text { Centri- } \\
\text { lobular } \\
\text { Emphysema } \\
(\%)\end{array}$} & \multirow{2}{*}{$\underset{(\%)}{\text { Panlobular }} \underset{\text { Emphysema }}{ }$} & \multirow{2}{*}{$\begin{array}{c}\text { Bronchial } \\
\text { Mucous } \\
\text { Gland Size } \\
(\%)\end{array}$} & \multirow{2}{*}{$\begin{array}{c}\text { Small } \\
\text { Airways } \\
\text { Lumen } \\
(\%)\end{array}$} & \multirow{2}{*}{$\begin{array}{c}\text { Thick-walled } \\
\text { Peripheral } \\
\text { Lung } \\
\text { Vessels } \\
(\%)\end{array}$} & \multirow{2}{*}{$\begin{array}{c}\text { Acute } \\
\text { Bronchitis }\end{array}$} & \multirow{2}{*}{$\begin{array}{l}\text { Pulmonary } \\
\text { Thrombo- } \\
\text { embolism }\end{array}$} \\
\hline & Left & Right & & & & & & & & & \\
\hline $\begin{array}{r}9 \\
15 \\
19 \\
20 \\
50 \\
68 \\
72 \\
74\end{array}$ & $\begin{array}{l}156 \\
186 \\
172 \\
212 \\
104 \\
167 \\
125 \\
120\end{array}$ & $\begin{array}{r}60 \\
43 \\
105 \\
114 \\
55 \\
80 \\
60 \\
50\end{array}$ & $\begin{array}{l}2 \cdot 6 \\
4 \cdot 3 \\
1 \cdot 6 \\
1 \cdot 9 \\
1 \cdot 9 \\
2 \cdot 1 \\
2 \cdot 1 \\
2 \cdot 4\end{array}$ & $\begin{array}{l}40^{*} \\
18^{*} \\
70 \\
47 \\
64 \\
40^{*} \\
66^{*} \\
28^{*}\end{array}$ & $\begin{array}{r}38 \\
18 \\
4 \\
8 \\
26 \\
8 \\
0 \\
6\end{array}$ & $\begin{array}{r}2 \\
0 \\
66 \\
39 \\
38 \\
32 \\
66 \\
22\end{array}$ & $\begin{array}{l}21 \cdot 8 \\
23 \cdot 0 \\
16 \cdot 0 \\
23 \cdot 0 \\
20 \cdot 1 \\
14 \cdot 0 \\
28 \cdot 4 \\
14 \cdot 3\end{array}$ & $\begin{array}{l}1.40 \\
0.57 \\
1.59 \\
0.76 \\
1.01 \\
1.28 \\
0.96 \\
0.72\end{array}$ & $\begin{array}{r}19.2 \\
6.5 \\
34.8 \\
19.9 \\
28.8 \\
10.6 \\
17.2 \\
15.4\end{array}$ & $\begin{array}{l}+ \\
+ \\
+ \\
+ \\
+ \\
+ \\
+ \\
+\end{array}$ & $\begin{array}{l}- \\
\pm \\
\pm \\
\pm \\
-\end{array}$ \\
\hline $\begin{array}{c}\text { Mean } \\
\text { SD }\end{array}$ & $\begin{array}{r}155 \cdot 2 \\
34 \cdot 2\end{array}$ & $\begin{array}{l}70 \cdot 8 \\
26 \cdot 2\end{array}$ & $\begin{array}{l}2 \cdot 28 \\
0.79\end{array}$ & $\begin{array}{l}46 \cdot 6 \\
18 \cdot 8\end{array}$ & $\begin{array}{l}13 \cdot 5 \\
12.9\end{array}$ & $\begin{array}{l}33 \cdot 1 \\
25 \cdot 0\end{array}$ & $\begin{array}{r}20 \cdot 0 \\
5 \cdot 0\end{array}$ & $\begin{array}{l}1.04 \\
0.35\end{array}$ & $\begin{array}{r}19 \cdot 0 \\
8 \cdot 0\end{array}$ & & \\
\hline
\end{tabular}

${ }^{1}$ Mean of right and left lungs.

T A B L E V I

COMPARISON OF MEAN VALUES OF PATHOLOGICAL PARAMETERS IN THE TWO GROUPS ( $t$ TEST)

\begin{tabular}{l|ccc}
\hline \multicolumn{1}{c|}{ Parameter } & $t$ & P & \\
\hline Left ventricular weight & 2.54 & $<0.02$ & Significant \\
Right ventricular weight & 4.78 & $<0.001$ & Significant \\
LV:RV ratio & 2.70 & $<0.02$ & Significant \\
Total amount of emphysema & 0.21 & $>0.80$ & \\
Centrilobular emphysema & 0.43 & $>0.60$ & \\
Panlobular emphysema & 0.01 & $>0.90$ & \\
Bronchial mucous gland size & 1.58 & $>0.10$ & \\
Small airways lumen & 1.46 & $>0.10$ & \\
Thick-walled peripheral lung & 0.70 & $>0.40$ & \\
vessels & 0.0 &
\end{tabular}

upper limit of normal $(190 \mathrm{~g})$ while being less than $225 \mathrm{~g}$. Of the 10 cases, nine were in the group which had clinical evidence of right ventricular failure.
Electrocardiographic changes were compared with the weight of the right ventricle. $P$ pulmonale was present in 16 patients of whom four (cases 4 , 50,72 , and 80) did not have right ventricular hypertrophy.

Five patients showed no $P$ pulmonale on the electrocardiogram but, of these, four had enlarged right ventricles at necropsy. Changes consistent with right ventricular hypertrophy in the chest leads were shown on 13 electrocardiograms, and only two of these (cases 9 and 50) did not have enlarged right ventricles. Of the eight patients who did not have electrocardiographic changes of right ventricular hypertrophy, three had normal right ventricular weights (cases 15,72 , and 74) and the remaining five did have an increased right ventricular weight. 
Chest radiographic changes were also compared with the findings in the lungs and heart found at necropsy. Emphysema was diagnosed on 15 radiographs, and all of these patients had pathological evidence of emphysema. However, there were two cases (21 and 30 ) in which $21 \%$ and $35 \%$ of emphysema respectively was present at necropsy and there were no significant signs of emphysema in the radiographs. Thirteen patients had radiological evidence of enlargement of the right ventricle and, of these, 10 had increased right ventricular weights and three had normal weights (cases 9, 50, and 74). Three patients (cases 20, 27 , and 34) had no radiological evidence of right ventricular enlargement but had an increased right ventricular weight at necropsy.

Twenty of the 21 patients had histological evidence of acute bronchitis and bronchiolitis at necropsy. Only case 27 did not show this change. Pneumonia was present histologically in four cases $(50,51,73$, and 80$)$. There was histological evidence of pulmonary thromboembolism in five cases $(19,20,50,66$, and 70$)$ out of the $21(24 \%)$.

\section{DISCUSSION}

Several studies have attempted to divide patients with obstructive airways disease into two distinct groups (Ogilvie, 1959; Richards, 1960; Fletcher et al., 1963; Burrows et al., 1964; Briscoe and Nash, 1965). One type, referred to as type B by Burrows et al. (1966), is said to have severe right ventricular failure, polycythaemia, and severely altered blood gases and is predominantly bronchitic. The other type, called type A, does not usually have right ventricular failure, polycythaemia or grossly disturbed blood gases and is predominantly emphysematous. Most of these studies did recognize that not all cases could be fitted into these two distinct groups, and several more recent studies have questioned whether this simplified division into two groups has any merit (Martin, Katsura, and Cochran, 1970; Park et al., 1970; Thurlbeck et al., 1970; Anderson et al., 1973). All the patients in the present series were bronchitics and would thus fall into either type $B$ or $X$ of the original Burrows classification. However, 15 of these patients had radiological evidence of emphysema, which is supposed to be a feature of type A disease. All the patients had severely disordered blood gases, but as these estimations were performed during the terminal phase of the disease, this measurement of respiratory failure is probably an indication of airways inflammation and blockage by mucous plugs rather than the nature of the disease in the steady state:o Eight of these patients did not have polycythaemiac which is stated to be relatively common in the type $B$ disease, and also eight of the patients hado never been in heart failure before the terminah episode. All the patients had significant amounts of emphysema pathologically, a feature in Bur? rows classification of type $A$ disease. The present $\vec{t}$ series of patients do not appear to fit easily intow the previously described groups, either clinically or pathologically. It has been suggested that heartw failure is more common in obstructive airways disease when emphysema is slight (Burrows et al. os 1966), and also that right ventricular failure is related to predominantly centrilobular rather thane to panlobular emphysema (Heath et al., 1968). Neither of these findings is confirmed in theo present study from which it appears that the amount and type of emphysema are not related $\mathrm{f}$ to the presence or absence of right ventricular failure. The group with right ventricular failure in the present study did die at a significantlye younger age than the group without failure, al-o though there was no difference in the total dura-D tion of the illness, and this confirms the finding of Bishop (1973) that right ventricular failure carries a poor prognosis in obstructive airways disease. The mean length of time that patients survived in right ventricular failure was three $\Rightarrow$ years in the present study and this is similar tog the findings of Ude and Howard (1971), whonoted that $68 \%$ of patients died within five yearso of the first attack of failure, and Bishop (1973) who found that $77 \%$ of patients with heart failureo were dead within four years.

A considerable group of patients die fromo chronic airways obstruction without developing pulmonary hypertension or right ventricularo hypertrophy (Bishop, 1973), and this study has shown no quantitative clinical or pathologicap difference, other than the size of the heart, be $\rightarrow$ tween this group and those who do develop righte ventricular failure.

Fulton (1953) suggested that the rate of progression of the disease may be of major importance $N$ as patients may die from airways obstruction before they have time to develop cardiac changes. No evidence of this is found in the present series of patients as the length of history of chest disease was not significantly different in those? with and without failure nor was there a differ-0 ence in the severity of the lung disease. As there is no fundamental difference in the nature or extent of the lung disease in those who die of heart failure and those who do not, the findingo 
of Lindsay and Read (1972) that there is a spectrum of vascular responsiveness in the pulmonary vessels to alveolar hypoxia is of interest. Patients with chronic airways obstruction who respond to the hypoxic stimulus develop pulmonary vessel contraction and eventually pulmonary hypertension, whereas non-responders do not behave in this manner and do not develop an elevated pulmonary artery pressure.

It has been stated that polycythaemia is more common in the 'bronchitic' type of patient who has cardiac failure than in the 'emphysematous' patient without failure (Ogilvie, 1959; Burrows et al., 1964). This is not confirmed in the present series where there was no significant difference between the number of patients with polycythaemia in the right ventricular failure group and the non-failure group. Nor was there a difference in the degree of polycythaemia, as measured by the haemoglobin and packed cell volume, between the two groups. These haematological measurements were made during the terminal phase of the illness, and this may reflect the effects of a recent severe hypoxic episode as well as longstanding moderately reduced oxygen levels. There was no confirmation of the finding of Millard and Reid (1974) of a relationship between the weight of the right ventricle and the packed cell volume.

This study has shown a good correlation between the presence of clinical right ventricular failure and right ventricular hypertrophy at necropsy. Other studies (Thurlbeck et al., 1970; Watanabe, Mitchell, and Renzetti, 1965) have noted similar correlations but, as in these series, there are exceptions. Three cases were present in which the right ventricle was enlarged but the patients had not been in clinical right ventricular failure. It appears from the present series that patients in right ventricular failure have developed an enlarged right ventricle but that some patients may develop right ventricular hypertrophy without subsequent heart failure (Thomas, 1972).

The electrocardiographic changes correlated reasonably well with the necropsy findings in the right ventricle. The presence or absence of $\mathbf{P}$ pulmonale proved to be a correct index of the presence or absence of right ventricular hypertrophy in 13 out of 21 cases $(62 \%)$, and similarly electrocardiographic signs of 'right ventricular hypertrophy' in the chest leads were correct in $66 \%$ of cases. This degree of accuracy in the electrocardiographic detection of an enlarged right ventricle is similar to that of other studies (Fulton, 1953; Rees, Thomas, and Rossiter, 1964;
Padmavati and Raizada, 1972). Cases where the electrocardiographic changes and the right ventricular weight do not correspond may well be due to the fact that overdistension of the right ventricle may cause changes in the electrocardiogram although there is no increase in the weight of the ventricular muscle (Burrows et al., 1964).

The radiographic diagnosis of emphysema proved to be accurate when compared to the necropsy findings, and in only two cases where there was a significant amount of emphysema at necropsy were radiological changes absent. In severe degrees of emphysema radiology has been shown to be a useful indicator of the presence of the disease (Thurlbeck, 1963; Burrows et al., 1966), but it is less reliable when only small amounts of emphysema are present (Thurlbeck et al., 1970). In the present series, where all the patients had severe airways obstruction, this high incidence of radiological emphysema would be expected due to overinflation of the lungs.

The radiographic estimation of the presence or absence of right ventricular enlargement was correct in 11 out of 17 cases $(65 \%)$ in the present study. There were three false positives and three false negatives in the series when the radiographic changes were compared to the actual weight of the right ventricle. Previous studies have shown a similar number of inaccuracies in chest radiographic estimation of the size of the right ventricle (World Health Organisation, 1961; Thurlbeck et al., 1970) and much of this is due to the increase in the radiographic size of the right ventricle being caused by dilatation rather than hypertrophy (World Health Organisation 1961; Cullen et al., 1970).

This study has also shown the possible importance of an episode of acute infection in the airways in precipitating death in patients with chronic airways obstruction. Only one of the patients in the series did not have acute bronchitis histologically at necropsy. Pneumonia, however, was not a particularly common event in the present series. The importance of infection and its association with episodes of heart failure, in chronic airways obstruction, was pointed out by Fulton (1953) and Simpson (1958), and both these authors stressed the point that this terminal infection may be overlooked by the clinician as the temperature of the patient is often normal. This proved to be the case in the present series where the clinicians stated that there was no evidence of acute respiratory infection during life in the majority of the patients. Episodes of acute 
bronchitis may precipitate right ventricular failure (Simpson 1958; Sweet et al., 1961) but in the present series it was present equally in the patients with and without right ventricular failure.

Pulmonary thromboembolism contributed to the death of five patients in the present series $(24 \%)$ but did not cause death in any of them. Few studies of the incidence of thromboembolic disease in chronic airways obstruction have been done but this percentage is similar to that of 29 found by Thurlbeck et al. (1970).

The finding of left ventricular hypertrophy at necropsy in patients with chronic airways obstruction has been noted by several authors (Michelson, 1960; Fluck, Chandraseker, and Gardner, 1966; Williams et al., 1968, Fishman, 1971; Edwards, 1974). The left ventricular weight was greater than normal in 10 patients in the present series but in only one of these was the increase great enough to be called true left ventricular hypertrophy. All except one of the patients who had enlarged left ventricles were in the group with right ventricular failure. These patients also had right ventricular hypertrophy, and this confirms previous findings of a correlation between the size of the right and left ventricles (Boushy et al., 1971; Edwards, Heath, and Harris, 1971). As muscle bands extend around both ventricles, hypertrophy of one is thought to cause concomitant hypertrophy of the other.

I wish to thank Drs. P. B. Anderson and P. Howard for their advice and cooperation, and Mrs. M. E. Jones for typing the manuscript.

\section{REFERENCES}

Anderson, P. B., Cayton, R. M., Holt, P. J., and Howard, P. (1973). Long-term oxygen therapy in cor pulmonale. Quarterly Journal of Medicine, 42, 563.

Bishop, J. M. (1973). Cardiovascular complications of chronic bronchitis and emphysema. Medical Clinics of North America, 57, 771.

Boushy, S. F., Aboumrad, M. H., North, L. B., and Helgason, A. H. (1971). Lung recoil pressure, airway resistance, and forced flows related to morphologic emphysema. American Review of Respiratory Disease, 104, 551.

Brenner, O. (1935). Pathology of the vessels of the pulmonary circulation. Pt. I. Archives of Internal Medicine, 56, 211.

Briscoe, W. A. and Nash, E. S. (1965). The slow space in chronic obstructive pulmonary disease. Annals of the New York Academy of Science, 121, 706.

Burrows, B., Fletcher, C. M., Heard, B. E., Jones, N. L., and Wootliff, J. S. (1966). The emphysematous and bronchial types of chronic airways obstruction. Lancet, 1, 830.
Burrows, B., Niden, A. H., Fletcher, C. M., ando Jones, N. L. (1964). Clinical types of chronic $\frac{\bar{\sigma}}{\sigma}$ obstructive lung disease in London and in Chicago. American Review of Respiratory Disease, 90, 14.

Ciba Guest Symposium Report (1959). Terminology, ֶొ definitions, and classification of chronic pul- monary emphysema and related conditions.? Thorax, 14, 286.

Cullen, J. H., Kaemmerlen, J. T. Daoud, A., and $\overrightarrow{\mathcal{\omega}}$ Katz, H. L., (1970). A prospective clinical-pathologic study of the lungs and heart in chronic $x$ obstructive lung disease. American Review of Respiratory Disease, 102, 190.

De Gruchy, G. C. (1964). Clinical Haematology in் Medical Practice, 2nd edition, p. 35. Blackwell,, $\mathfrak{\omega}$ Oxford.

Dunnill, M. S. (1962). Quantitative methods in the study of pulmonary pathology. Thorax, 17, 320.

Dunnill, M. S. (1968). Quantitative methods in histology. In Recent Advances in Clinical Path-通 ology, Series, 5, edited by S. C. Dyke, p. $405 . \overline{3}$ Churchill, London.

Dunnill, M. S., Massarella, G. R., and Anderson, $\vec{\varphi}$ J. A. (1969). A comparison of the quantitative anatomy of the bronchi in normal subjects, in? status asthmaticus, in chronic bronchitis, and in emphysema. Thorax, 24, 176.

Edwards, C. W. (1974). Left ventricular hypertrophy in emphysema. Thorax, 29, 75.

Edwards, C. W., Heath, D., and Harris, P. (1971). The carotid body in emphysema and left ventriculare hypertrophy. Journal of Pathology, 104, 1.

Fishman, A. P. (1971). The left ventricle in 'chronic bronchitis and emphysema'. New England Journal? of Medicine, 285, 402 .

Fletcher, C. M., Hugh-Jones, P., McNicol, W. M. and Pride, N. B. (1963). The diagnosis of pul-0 monary emphysema in the presence of chronich bronchitis. Quarterly Journal of Medicine, 32, 33×

Fluck, D. C., Chandraseker, R. G., and Gardner F. V. (1966). Left ventricular hypertrophy in chronic bronchitis. British Heart Journal, 28, 92.0

Fulton, R. M. (1953). The heart in chronic pulmonary disease. Quarterly Journal of Medicine, 22, 43 . 윽

Fulton, R. M., Hutchinson, E. C., and Jones, A. MD (1952). Ventricular weight in cardiac hypertrophy을 British Heart Journal, 14, 413.

Heard, B. E. (1958). A pathological study of emphys sema of the lungs with chronic bronchitis N Thorax, 13, 136.

Heard, B. E. (1969). Pathology of Chronic Bronchitis and Emphysema, p. 8. Churchill, London.

Heath, D., Brewer, D., and Hicken, P. (1968). Cor Pulmonale in Emphysema: Mechanisms and Pathology, p. 44. Thomas, Springfield, Illinois.

Hill, A. B. (1971). Principles of Medical Statistics $\frac{T}{0}$ 9th edition, p. 143. Lancet, London.

Hunter, C., Barer, G. R., Shaw, J. W., and Clegg E. G. (1974). Growth of the heart and lungs im hypoxic rodents: a model of human hypoxic 
disease. Clinical Science and Molecular Medicine, 46, 375.

Laënnec, R. T. H. (1819). De l'A uscultation Médiate. Brosson and Chaudé, Paris. English translation by J. Forbes (1830). S. Wood, New York.

Lindsay, D. A. and Read, J. (1972). Pulmonary vascular responsiveness in the prognosis of chronic obstructive lung disease. American Review of Respiratory Disease, 105, 242.

Martin, C. J., Katsura, S., and Cochran, T. H. (1970). The relationship of chronic bronchitis to the diffuse obstructive pulmonary syndrome. American Review of Respiratory Disease, 102, 362.

Matsuba, K. and Thurlbeck, W. M. (1971). The number and dimensions of small airways in nonemphysematous lungs. American Review of Respiratory Disease, 104, 516.

Matsuba, K. and Thurlbeck, W. M. (1972). The number and dimensions of small airways in emphysematous lungs. American Journal of Pathology, 67, 265.

Michelson, N. (1960). Bilateral ventricular hypertrophy due to chronic pulmonary disease. Diseases of the Chest, 38, 435.

Millard, J. and Reid, L. (1974). Right ventricular hypertrophy and its relationship to chronic bronchitis and emphysema. British Journal of Diseases of the Chest, 68, 103.

Ogilvie, C. (1959). Patterns of disturbed lung function in patients with chronic obstructive vesicular emphysema. Thorax, 14, 113.

Padmavati, S. and Raizada, V. (1972). Electrocardiogram in chronic cor pulmonale. British Heart Journal, 34, 658.

Park, S. S., Janis, M. Shim, C. S., and Williams, M. H., Jr. (1970). Relationship of bronchitis and emphysema to altered pulmonary function. American Review of Respiratory Disease, 102, 927.

Rees, H. A., Thomas, A. J., and Rossiter, C. (1964). The recognition of coronary heart disease in the presence of pulmonary disease. British Heart Journal, 26, 233.
Restrepo, G. and Heard, B. E. (1963). The size of the bronchial glands in chronic bronchitis. Journal of Pathology and Bacteriology, 85, 305.

Richards, D. W. (1960). Pulmonary emphysema: etiologic factors and clinical forms. Annals of Internal Medicine, 53, 1105.

Simpson, T. (1958). Chronic bronchitis and emphysema. Tubercle (London), 39, 307.

Sweet, H. C., Wyatt, J. P., Fritsch, A. J., and Kinsella, P. W. (1961). Panlobular and centrilobular emphysema: correlation of clinical findings with pathologic patterns. Annals of Internal Medicine, $55,565$.

Thomas, A. J. (1972). Chronic pulmonary heart disease. British Heart Journal, 34, 653.

Thurlbeck, W. M. (1963). A clinico-pathological study of emphysema in an American hospital. Thorax, 18, 59.

Thurlbeck, W. M., Henderson, J. A., Fraser, R. G., and Bates, D. V. (1970). Chronic obstructive lung disease. Medicine (Baltimore), 49, 81.

Ude, A. C. and Howard, P. (1971). Controlled oxygen therapy and pulmonary heart failure. Thorax, 26, 572.

Watanabe, S., Mitchell, M., and Renzetti, A. D., Jr. (1965). Correlation of structure and function in chronic pulmonary emphysema. American Review of Respiratory Disease, 92, 221.

Williams, J. F., Jr., Childress, R. H., Boyd, D. L., Higgs L. M., and Behnke, R. H. (1968). Left ventricular function in patients with chronic obstructive pulmonary disease. Journal of Clinical Investigation, 47, 1143.

World Health Organisation (1961). Chronic cor pulmonale: report of an expert committee. World Health Organization, Technical Report Series, 213, 12.

Requests for reprints to: Dr. K. W. M. Scott, Pathology Department, The Royal Hospital, Wolverhampton. 